\title{
THE INCREASE IN THICKNESS UNIFORMITY OF FILMS OBTAINED BY MAGNETRON SPUTTERING WITH ROTATING SUBSTRATE
}

\author{
Golosov D. ${ }^{a, *}$, Melnikov S. ${ }^{a}$, Zavadski S. ${ }^{a}$, Kolos V. ${ }^{b}$, Okojie J. ${ }^{a}$ \\ ${ }^{a}$ Belarusian State University of Informatics and Radioelectronics, P. Brovka, 6, 220013, Minsk, Belarus \\ b JSC "INTEGRAL", I.P. Kazintsa, 121A, 220108 Minsk, Belarus \\ * dmgolosov@gmail.com
}

\begin{abstract}
The titanium thin films obtained by magnetron sputtering with the rotating substrate at different distances between the substrate and magnetron centers were studied with regard to the uniformity of the film thickness distribution. On the basis of the experimental data obtained, the model for the magnetron film deposition during substrate rotation was developed. The analysis of the simulation results shows that the model error is not greater than $10 \%$.
\end{abstract}

Keywords: magnetron sputtering, deposition rate, film thickness, nonuniformity.

\section{Introduction}

Now magnetron sputtering is a more frequently used method for the deposition of thin films 1 . In microelectronic this method is utilized not only for the formation of metalization films, but also for the preparation of the oxide and nitride films, e.g., ferroelectric, high-temperature superconductors, magnetoresistive thin films, etc [2, 3]. It should be noted that the development of microelectronic implies the constant decrease in the component dimensions. This results in the proportional decrease in the thickness of the deposited films. At the same time, the diameter of the silicon wafer increase to $300 \mathrm{~mm}$, and in the nearest future they will grow to $450 \mathrm{~mm}$. Therefore, for the thin film deposition the methods of the individual substrate treatment are applied. For this reason, the deposited thin films of high thickness uniformity are of great demand.

During magnetron sputtering in order to increase the uniformity of the film thickness distribution the sputtering area is increased in most cases. To deposit films onto the large substrates, the magnetrons equipped with several sputtering areas or magnetrons of complex shape were designed [4]. However, it implies the fabrication of the large targets, but sometimes it cannot be achieved. For the fabrication of component and multicomponent targets the cost for the initial materials and fabrication itself can be very high. The large target can also crack during fabrication and operation.

Among others magnetron sputtering techniques, the application of rotating substrates is one of the relatively simple methods to increase the uniformity of the deposited layers. For the small sputtering areas this method allows for obtaining relatively uniform in film thickness even onto the substrates that outmeasure the target in several times. However, often at designing of such systems the relative positioning of the magnetron and the substrate is selected em- pirically. Herewith, the peculiarities of the different sputtering materials do not take into account. The application of the simulations can significantly decrease the terms and costs for the development and exclude the mistakes during design of the equipment for the magnetron sputtering of thin films.

The majority of the modern methods for the calculations of the thickness of the deposited films are based on the principles suggested by Holland [5]. This model is based on the integration of sputtered flow from the each point of sputtered area. In the sequel this model was applied for the variety magnetron types and methods of substrate movement [6 9]. However, these models do not consider the peculiarities of the current density distribution in the sputtering area of magnetron, space distribution of sputtered atoms, and the data on the errors of these models are practically absent.

So, the work deals with the investigation of the thickness uniformity of the deposited films obtained by magnetron sputtering onto the rotating substrate in order to develop the method for computation of the systems.

\section{Experimental}

Schematic diagram of the magnetron sputtering system with rotating substrate is shown in Figure 1. The chamber of the vacuum setup VU-1 was equipped with magnetron sputtering system with target of diameter of $80 \mathrm{~mm}$ and end-Hall ion source. The ion surface pretreatment was carried out by ion source. In the experiments substrate holder rotating rate was kept constant of about $24.5 \mathrm{rpm}$. The substrate area was located at the distance of $100 \mathrm{~mm}$ from the target surface. The substrate-target distance was controlled by the magnetron shift.

During the experiments, the substrate from monocrystalline silicon wafer $\operatorname{Si}(100)$ with the diameter of $150 \mathrm{~mm}$ was placed on the rotating substrate 
holder. The chamber of the vacuum setup was evacuated to the base pressure of $10^{-3} \mathrm{~Pa}$ and in order to increase the film adhesion then ion beam cleaning of the substrates was carried out. For this, high-purity argon gas was introduced into the ion source up to the working pressure of $10^{-2} \mathrm{~Pa}\left(Q_{A r}=6 \mathrm{sccm}\right)$. The cleaning took place at rotating substrate. All experiments were carried out at a constant cleaning time of $3 \mathrm{~min}$, discharge voltage of $240 \mathrm{~V}$, and discharge current of $3.5 \mathrm{~A}$.

For the sputtering of Ti target (99.9\%) the magnetron sputtering system MARS.080.011 was used. The working gas was introduced into the gas distribution system. The gas flow was of about $35 \mathrm{sccm}$. The pressure was at about $0.06 \mathrm{~Pa}$ in the chamber. During depositing process the discharge current was kept constant and was about $2.0 \mathrm{~A}$; discharge voltage was $360-375 \mathrm{~V}$ and depositing time was $180 \mathrm{~s}$. The thickness uniformity of the deposited films was determined by optical interferometric profilometer POI-s08.

\section{Results and discussion}

The processes of the deposition of thin films onto the rotating substrate during magnetron sputtering of Ti target were studied. The profile of the thickness distribution was obtained at different shift of the substrate center relative to the target center (Figure 2). At discharge current of $2.0 \mathrm{~A}$ the deposition rate was about $1.1 \mathrm{~nm} \mathrm{~s}^{-1}$ on the magnetron axis and about $0.8 \mathrm{~nm} \mathrm{~s}^{-1}$ on the substrate edge. With increasing distance between the substrate and target centers, the deposition rate decreased, but at the same time the uniformity of the layers increased.

On the basis of the data obtained the model for magnetron deposition of the films onto the rotating substrate was developed. In Figure 3 the schematic diagram of the model is shown. The rotating substrate is placed on the axis of vacuum chamber. The magnetron sputtering system is positioned at the bottom of the chamber, in such a way, that the target surface is at the distance $h$ from the substrate surface and is parallel to it. The magnetron center is not coincident with that of the substrate. The coordinates for magnetron center are $\left(x_{m} ; y_{m}\right)$.

The deposition rate $V\left(r_{2}, t\right)$ at the time $t$ of the substrate surface point situated at the distance $r_{2}$ from the substrate center can be computed by Eq.(1)

$V\left(r_{2}, t\right)=\frac{1}{\pi} \int_{R_{\min }}^{R_{\max }} \int_{0}^{2 \pi}\left(\frac{r_{1} v_{t}\left(r_{1}\right) \cos ^{2} \varphi\left(r_{1}, r_{2}, \theta, t\right)}{l^{2}\left(r_{1}, r_{2}, \theta, t\right)}\right) d r_{1} d \theta$

where $R_{\min }$ and $R_{\max }$ are the minimum and maximum radii of erosion area; $\varphi$ is sputtering and condensation angle; $\theta$ is polar angle; $l$ the distance from the sputtering point to the condensation point; $v_{1}\left(r_{1}\right)$ the sputtering rate of the target material in the radius $r_{1}$

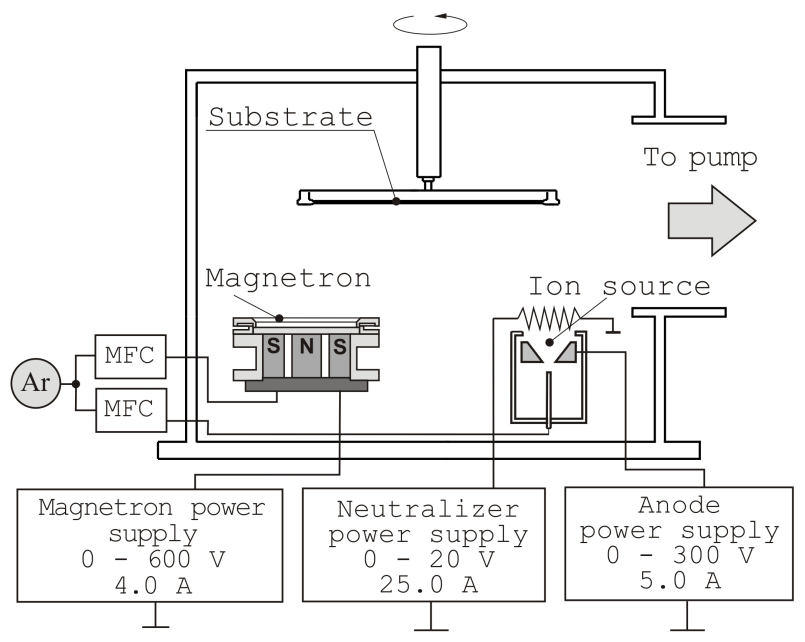

Figure 1. Schema of the magnetron sputtering setup with rotating substrate.

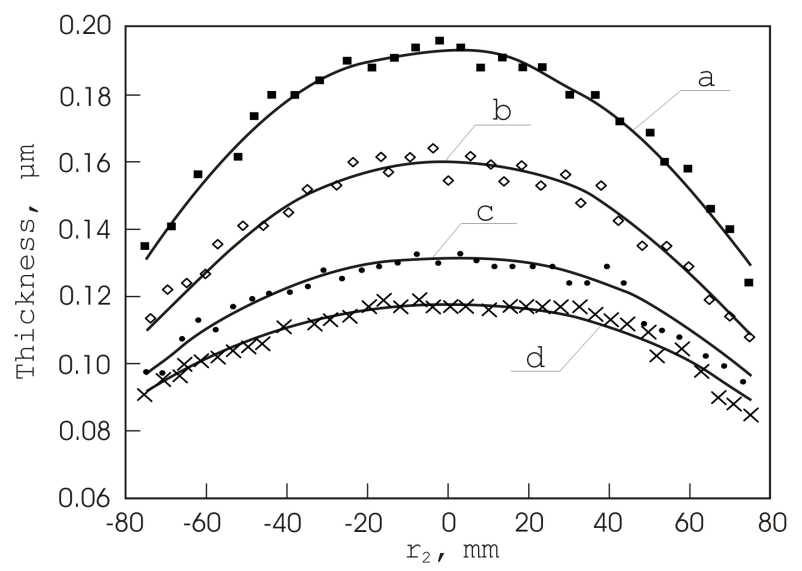

Figure 2. The profiles of the thickness distribution of the Ti films obtained at different shift from target center to that of magnetron: $a-0 \mathrm{~mm} ; b-30 \mathrm{~mm}$; $c-54 \mathrm{~mm} ; \mathrm{d}-75 \mathrm{~mm}$.

$$
v_{t}\left(r_{1}\right)=Y \frac{j_{t}\left(r_{1}\right) A}{N_{A} e \rho(1+\gamma)} .
$$

where $Y$ is the sputtering yield of the target material atoms; $e$ is the electron charge; $j_{t}\left(r_{1}\right)$ the discharge current density in the radius $r_{1} ; A$ atomic mass of the sputtering material; $\rho$ the density of target; $\gamma$ the coefficient of ion-electron emission of the target material; $N_{\mathrm{A}}$ Avogadro's number.

The distribution of the discharge current density $j_{t}\left(r_{1}\right)$ was fitted by double-Gaussian fit. In this distribution the real parameters of sputtering area and discharge current are used so this model allows us to formulate mathematically the distribution of the density of ion current with high accuracy. For this simulation the profile of distribution of ion current is set analytically by the following equation

$$
j_{t}\left(r_{1}\right)=\frac{I_{t}}{\pi\left(R_{\max }+R_{\min }\right)} \frac{f\left(r_{1}\right)}{\int_{0}^{\infty} f\left(r_{1}\right) d r_{1}}
$$




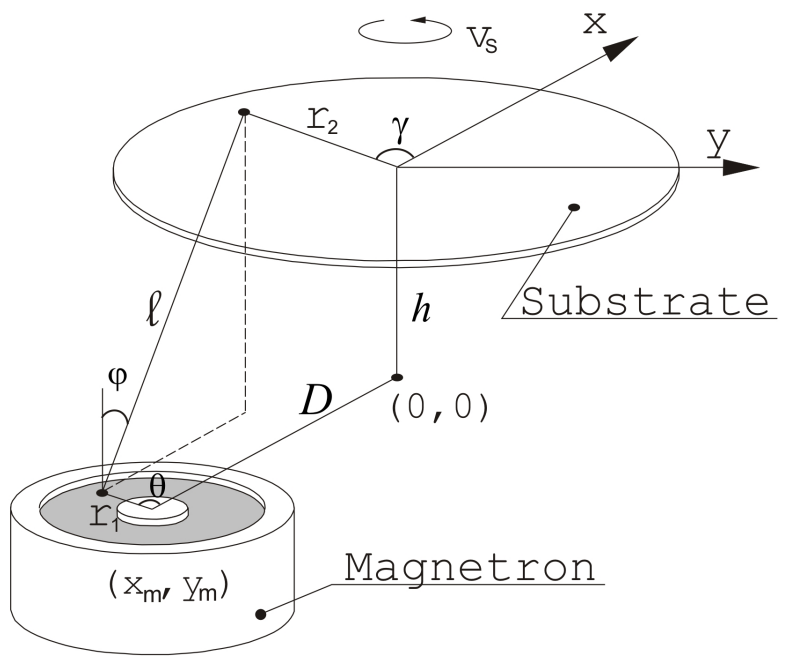

Figure 3. Schematic diagram of the magnetron sputtering system with rotating substrate.

where $I_{t}$ is magnetron discharge current; $f\left(r_{1}\right)$ the distribution function that is double semi-Gaussian

$$
\begin{gathered}
f\left(r_{1}\right)=\left\{\begin{array}{c}
\frac{1}{\sqrt{2 \pi} \sigma} \exp \left[-\frac{\left(r_{1}-\bar{R}\right)^{2}}{2 \sigma_{1}^{2}}\right] \text { for } r_{1} \leq \overline{\mathrm{R}} \\
\frac{1}{\sqrt{2 \pi} \sigma} \exp \left[-\frac{\left(r_{1}-\bar{R}\right)^{2}}{2 \sigma_{2}^{2}}\right] \text { for } r_{1}>\overline{\mathrm{R}}
\end{array}\right. \\
\sigma_{1}=\frac{\bar{R}-R_{\min }}{3} \quad \sigma_{2}=\frac{R_{\max }-\bar{R}}{3} \quad \sigma=\frac{R_{\max }-R_{\min }}{6},
\end{gathered}
$$

where $\bar{R}$ is maximum radius of erosion area. At the same time the distribution function must satisfy the following equation

$$
\int_{-\infty}^{\infty} j_{t}\left(r_{1}\right) d r_{1}=I_{t}
$$

During the process time $t_{d e p}$ the thickness of the deposited film of the point located in radius $r_{2}$ from the substrate center can be described by

$$
W\left(r_{2}\right)=\int_{0}^{t_{d e p}} V\left(r_{2}, t\right) d t
$$

The space distribution of the sputtering atoms is believed to follow the cosine distribution in the Eq. (1). However, Ti belongs to the materials for which the emission maximum during sputtering does not coincide with the direction of the normal to the surface (Figure 4] 10]. Therefore, for simulate the space distribution of the sputtered Ti particles the following function was applied

$$
F(\varphi)=\frac{\cos \varphi}{\alpha^{2} \sin ^{2} \varphi+\cos ^{2} \varphi} .
$$

where $\alpha$ is the coefficient on which the shape of the deposited particles depends. If $\alpha>1$ the function

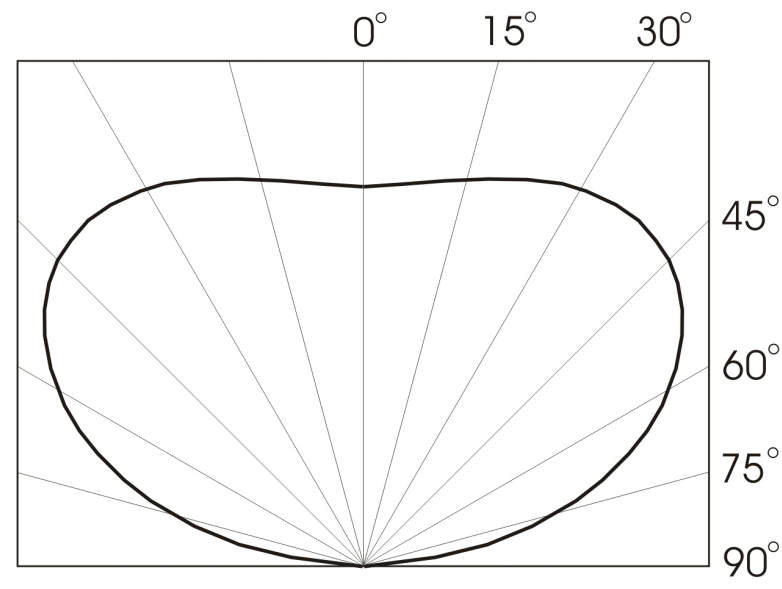

Figure 4. The space distribution of the sputtered particles during magnetron sputtering of Ti [10].

has the shape of over cosine distribution, if $\alpha<1$ has under cosine distribution, and if $\alpha=1$ the function has usual cosine distribution.

The distance from sputtering point to that of condensation and sputtering angle can be determined from geometry constructions. So the coordinates of the sputtering point at the target surface during integration can be computed as

$$
\begin{gathered}
x_{t}\left(r_{1}, \theta\right)=r_{1} \cos (\theta)+x_{m}, \\
y_{t}\left(r_{1}, \theta\right)=r_{1} \sin (\theta)+y_{m} .
\end{gathered}
$$

The angular rotation rate of the surface is

$$
v_{s}=2 \pi \frac{N_{s}}{60},
$$

where $N_{s}$ is the substrate rotation rate in rpm. The rotation angle of the substrate at the time point $t$ is

$$
\gamma(t)=v_{s} t
$$

Then, the coordinates for surface point in radius $r_{2}$ at the time point $t$ can be determined from

$$
\begin{gathered}
x_{s}\left(r_{2}, t\right)=r_{2} \cos (\gamma(t)), \\
y_{s}\left(r_{2}, t\right)=r_{2} \sin (\gamma(t)) .
\end{gathered}
$$

The sputtering angle

$$
\varphi\left(r_{1}, r_{2}, \theta, t\right)=\arcsin \left(\frac{h}{l\left(r_{1}, r_{2}, \theta, t\right)}\right),
$$

where

$$
l=\sqrt{\left(x_{s}-x_{t}-x_{m}\right)^{2}+\left(y_{s}-y_{t}-y_{m}\right)^{2}+h^{2}} .
$$

Utilizing the profile of the distribution of film thickness at different distance between centers of the substrate and magnetron, we calculated the uniformity of the film thickness (Figure 5). During calculations 


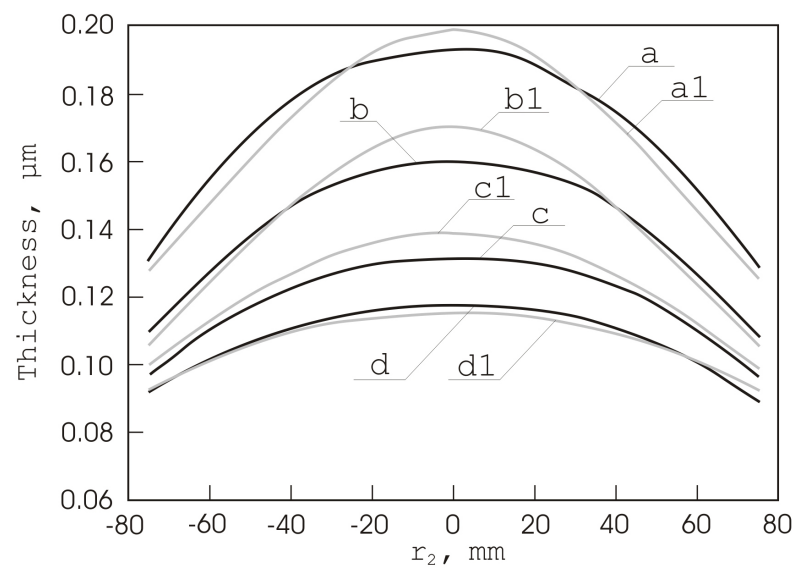

Figure 5. The computed $\left(a_{1}, b_{1}, c_{1}, d_{1}\right)$ and experimental $(a, b, c, d)$ profiles of the thickness distribution of Ti film at different shift from target center to that of magnetron: $a, a_{1}-0 \mathrm{~mm} ; b, b_{1}-30 \mathrm{~mm} ; \mathrm{c}, c_{1}-$ $54 \mathrm{~mm} ; \mathrm{d}, d_{1}-75 \mathrm{~mm}$.

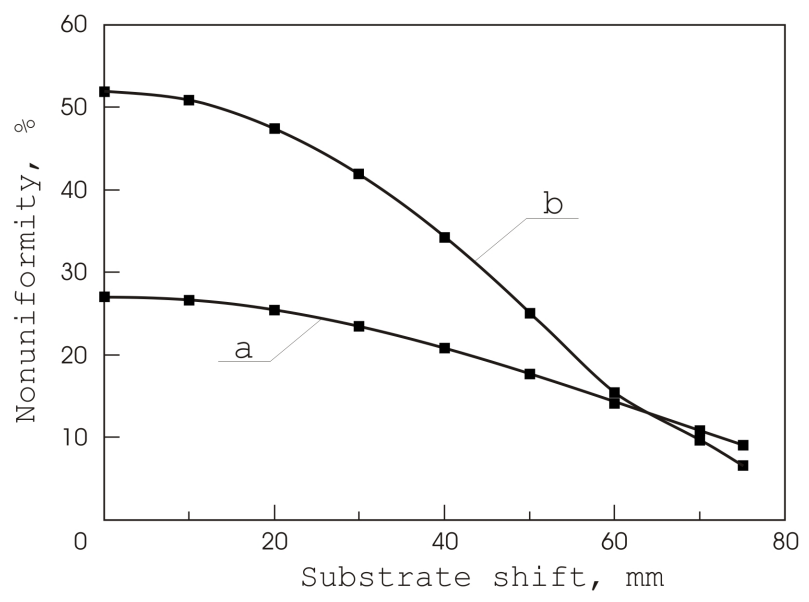

Figure 6. The dependence of nonuniformity of the Ti film thickness on the shift distance between centers of substrate and magnetron at different substrate-target distance: $a-100 \mathrm{~mm} ; b-60 \mathrm{~mm}$.

we used the following parameters: target material $\mathrm{Ti}$, sputtering yield $Y_{\mathrm{Ti}}=0.221, \alpha=0.55, \gamma_{\mathrm{Ti}}=0.01$, $\rho_{\mathrm{Ti}}=4.54 \mathrm{~g} \mathrm{~cm}^{-3}, A_{\mathrm{Ti}}=47.87$ a.u., $R_{\min }=4.5 \mathrm{~mm}$, $R_{\text {max }}=32.5 \mathrm{~mm}, \bar{R}=19.5 \mathrm{~mm}$. The distance targetssubstrate was of $100 \mathrm{~mm}$. The substrate rotation rate was of $24.5 \mathrm{rpm}$, the discharge current of $2.0 \mathrm{~A}$, depositing time $180 \mathrm{~s}$.

For comparison the experimental results for film thickness distribution are also shown in Figure 5 As can be seen, the computed profiles agree with experimental data. The error does not exceed $10 \%$. So, the developed model can be used for forecasting of the film thickness distribution at magnetron sputtering onto the rotating substrate.

Utilizing the developed model, we calculated the uniformity of film thickness in $\varnothing 150 \mathrm{~mm}$ substrate at different distances between centers of the substrate and magnetron (Figure 60. As can be seen, with increasing distance the nonuniformity is decreasing

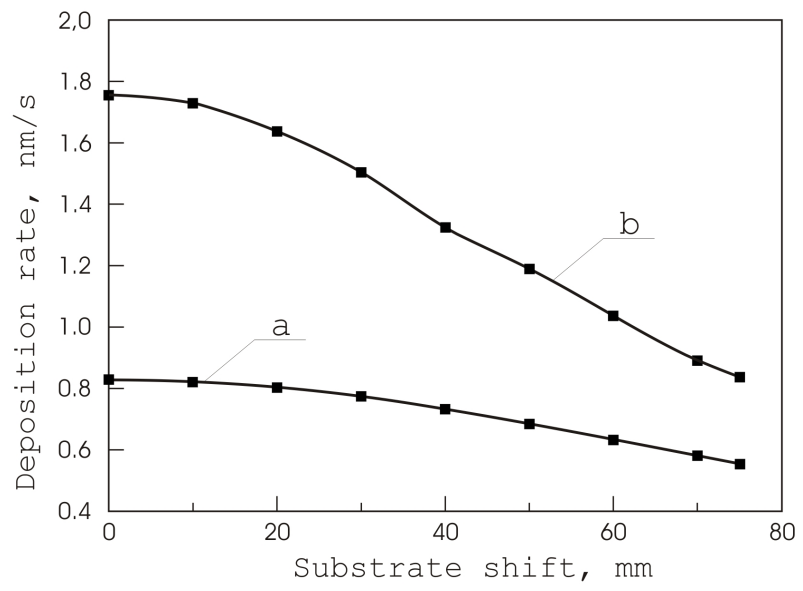

Figure 7. The dependence of the average deposition rate of Ti film on the shift distance between centers of substrate and magnetron at different substrate-target distance: $a-100 \mathrm{~mm} ; b-60 \mathrm{~mm}$.

and when the shift is $75 \mathrm{~mm}$ the films nonuniformity less than $10 \%$ can be obtained. However, it implies the decrease in the average depositing rate in about 2 times (Figure 7). The decrease in the deposition rate can be compensated by the decrease in the distance target-substrate (see Fig. 7, curve b). However, it results in the significant increase in the nonuniformity (see Fig. 6, curve b), and it can be clearly seen at small distance between target.

Besides, this model does not take into account the thermalization effect for the sputtered atoms. With increasing shift distance, the distance from sputtering point to condensation one also increases. At distance shift of $75 \mathrm{~mm}$ the maximum distance from sputtering point to the condensation one can reach $150 \mathrm{~mm}$. At the same time, the probability of thermalization of sputtered flow and its interaction with atoms of residual gases increases [11.

As is known, the direct path length of the sputtered atoms depends on the nature of the sputtered material and working pressure [11]. Therefore, for the determination of the process configuration the working pressure, distance substrate-target and shift distance between centers of substrate and magnetron should be chosen, in such a way, that the distance of the direct path for the sputtered atoms exceed the maximum distance from the point of the sputtering to the condensation point.

\section{Conclusions}

The titanium thin films obtained by magnetron sputtering with the rotating substrate were studied. It was established that the increase in the shift distance between the centers of substrate and target allows for improving the uniformity of film thickness at decreasing deposition rate.

On the basis of the experimental data obtained, the model for the magnetron layer deposition during substrate rotation was developed. The error does not 
exceed $10 \%$. So, the developed model can be used for forecasting of the film thickness distribution at magnetron sputtering onto the rotating substrate.

\section{References}

[1] Pauleau Y. Materials surface processing by directed energy techniques. Vol. 1: Fundamental algorithms. Elsevier ltd, 2006.

[2] Golosov D. Ferroelectric properties of niobium-doped strontium bismuth tantalate films. Physics of the Solid State, 58(1):50-54, 2016.

[3] Golosov D. Physical and electrical properties of yttria-stabilized zirconia thin films prepared by radio frequency magnetron sputtering. Acta Polytecnica, 53(2):155-159, 2013.

[4] P. Frach. The double ring process module-a tool for stationary deposition of metals, insulators and reactive sputtered compounds. Surf. Coat. Technol, 90:75-81, 1997.

[5] Holland L. Deposition of Thin Films. Chapman \& Hall, 1956.

[6] Chunlin Fu. The thickness uniformity of films deposited by magnetron sputtering with rotation and revolution. Surface E Coatings Technology, 200:3687-3689, 2006.

[7] Du X. S. Quantitative evaluation of film thickness uniformity: Application to off-axis magnetron source onto a rotating substrate. Journal of Vacuum Science $\mathbb{E}$ Technology A, 25(2):215-220, 2007.

[8] Jiang C.Z. Uniform film in large areas deposited by magnetron sputtering with a small target. Surface $\mathbb{E}$ Coatings Technology, 229:222-225, 2013.

[9] Wang Tao. Analysis of film thickness for magnetron sputtering system with more than one workbench. Proc. SPIE 7506, 2009 International Conference on Optical Instruments and Technology: Optical Systems and Modern Optoelectronic Instruments, 75062B (1 December 2009); doi: 10.1117/12.837841, 2009.

[10] Martynenko Y. Angular distribution of atoms during the magnetron sputtering of polycrystalline targets. Technical Physics, 57(4):439-444, 2012.

[11] Golosov D. Simulation of the film thickness distribution for magnetron sputtering systems with drum-type substrate holders. Fizika $i$ himiya obrabotki materialov (in russian), (4):28-37, 2012. 